УДК 534.21

\title{
МАТЕМАТИЧЕСКОЕ МОДЕЛИРОВАНИЕ УЛЬТРАЗВУКОВОГО ИЗЛУЧАТЕЛЯ РЕЗОНАНСНОГО ТИПА ДЛЯ ПОДГОТОВКИ ВЫСОКОВЯЗКОЙ НЕФТИ К ТРАНСПОРТУ
}

\author{
Азин Антон Владимирович1, \\ antonazin@niipmm.tsu.ru
}

Богданов Евгений Петрович2, epbogdanov@mail.ru

\author{
Марицкий Николай Николаевич ${ }^{1}$, \\ nnmar@niipmm.tsu.ru
}

\section{Пономарев Сергей Александрович 1 , doc.sergeyponomarev@gmail.com}

\author{
Рикконен Сергей Владимирович1, \\ rikk2@yandex.ru \\ 1 Национальный исследовательский Томский государственный университет, \\ Россия, 634050, г. Томск, пр. Ленина, 36. \\ 2 Национальный исследовательский Томский политехнический университет, \\ Россия, 634050, г. Томск, пр. Ленина, 30.
}

\begin{abstract}
Актуальность данного исследования обусловлена тем, что для повышения эфффективности (производительности) ультразвукового метода подготовки высоковязкой нефрти к транспорту необходимо полностью учитывать все особенности конструкции ультразвукового излучателя с целью дальнейшего полного энергетического согласования колебательной системы «излучатель-нагрузка».

Цель: разработка математической модели ультразвукового излучателя резонансного типа с учетом влияния деформации корпуса излучателя и колебаний многослойного пьезоактюатора на его амплитудно-частотные характеристики с целью получения суммарных энергетических характеристик системы.

объекты: конструкция ультразвукового излучателя резонансного типа, амплитудно-частотные характеристики.

Методы: математическое моделирование ультразвукового излучателя резонансного типа, учитьвающее колебательные процессы инерционности подвижных частей конструкции, подвижность части корпуса излучателя и колебательные процессы многослойного пьезоактюатора.

Результаты. Математическая модель позволяет рассчитать амплитудно-частотные характеристики колебательной системы с учетом деформаций корпуса ультразвукового излучателя и колебательных процессов многослойного пьезоактюатора, определиться с резонансными частотами системы, выбрать конструкцию корпуса излучателя и пьезопакета. Pезультаты теоретических расчетов хорошо согласуются с экспериментальными данными, отклонение составляет не более $15 \%$.

Выводы. Анализ результатов проведенного исследования показал, что для получения достоверной информации по работе ультразвукового излучателя резонансного типа при аналитических расчетах необходимо учитывать влияние жесткости корпуса и внутренних колебаний многослойного пьезоактюатора. Деформации части корпуса ультразвукового излучателя за счет наличия смотровых окон увеличивают ускорение рабочей поверхности толкателя по сравнению с работой ультразвукового излучателя с абсолютно жестким корпусом, тем самым увеличивается коэффрициент преобразования электрической энергии в механическую. Математическая модель ультразвукового излучателя резонансного типа позволяет определить частоты колебательной системы, на которых наблюдается максимальный коэфффициент преобразования энергии, и оценить необходимую точность настройки частоты источника энергии.
\end{abstract}

\section{Ключевые слова:}

Ультразвуковое излучение, излучатель, коэфффициент преобразования, энергия, тепловые поля.

Ультразвуковое излучение (УЗ) позволяет осуществлять энергетическое воздействие на разнообразные среды и элементы конструкций с разными физическими свойствами - жидкими, твердыми. Для твердых веществ УЗ воздействие осуществляется за счет гистерезисного нагрева, и данный эффект используется в технике и медицине [1-5].

Метод широко используется на этапе тестирования, тренировки и выбраковки радиоэлектронного оборудования специального назначения (бортовое оборудование космических аппаратов) как бескон- тактный и неразрушающий метод контроля. Передаваемая энергия создает градиент температур и тепловые поля в электронной плате, тем самым вызывая напряженно-деформированное состояние, что позволяет оценить долговечность материалов и устройства в целом. Учитывая техническую сложность конечных электронных изделий и стоимость их разработки, факт использования метода УЗ излучения подтверждает свою применимость, полезность и значимость. Данные принципы положены в основу при разработке математической модели УЗ излучателя. 
При влиянии УЗ на жидкие вещества (вязкие углеводороды) энергетическое воздействие осуществляется нагревом, сдвиговыми напряжениями при акустическом течении, кавитацией [6]. Ультразвуковой технологией можно существенно повысить эффективность подготовки высоковязкой нефти к транспорту - существенно снизить вязкость и дегазировать нефть. Источником акустической энергии является УЗ излучатель. Количество передаваемой энергии в среду зависит от конструкции излучателя [7-9]. Есть ряд конструкций излучателей с разными коэффициентами преобразования электрической энергии в механическую при разных условиях согласования источника с нагрузкой $[1,10-12]$. От коэффициента преобразования и согласования излучателя с нагрузкой зависит конечный технологический эффект - разрушение надмолекулярной структуры нефти и температура нагрева элемента конструкции.

В работе предложена математическая модель УЗ излучателя резонансного типа. Модель позволяет учитывать колебательные процессы, происходящие в системе УЗ излучателя:

- колебания подсистемы «толкатель - упругость предварительного поджатия»;

- колебательная подсистема «корпус - упругость корпуса»;

- колебательная подсистема многослойного пьезоактюатора.

На рис. 1 представлен УЗ излучатель резонансного типа, выполненный на основе многослойного пьезоактюатора АПМ-2-7. УЗ излучатель резонансного типа состоит из: 1 - корпус излучателя; 2,6 - центраторы; 3 - многослойный пьезоактюатор АПМ-2-7; 4 толкатель; 5 - упругость; 7 - датчик силы; 8 - смотровые окна в корпусе излучателя; 9 - стальная щека; 10 - гайка предварительного поджатия, 11 - стопорная гайка.

Изучение режимов работы УЗ излучателя удобно проводить по частотным характеристикам механической колебательной системы, которые позволяют анализировать резонансные явления устройства.

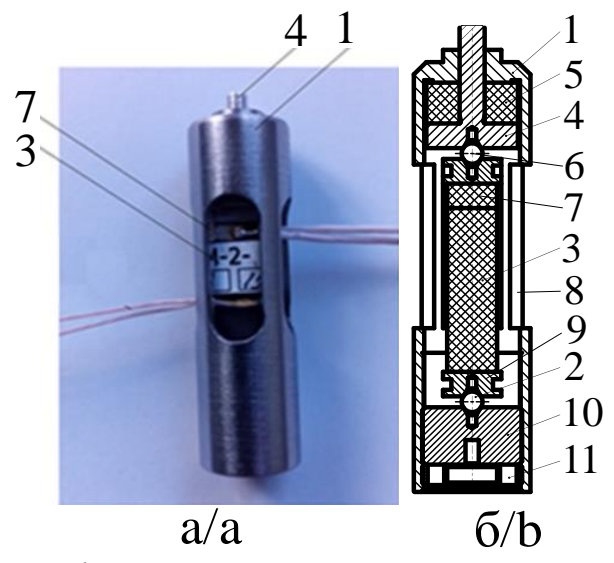

Pис. 1. Ультразвуковой излучатель резонансного типа: a) вариант изготовления; б) структурная схема

Fig. 1. Ultrasonic resonant emitter: a) manufacturing variant; b) structural design

Для аналитического определения амплитудночастотной характеристики (АЧХ) УЗ излучателя используется электрическая схема замещения, представленная на рис. 2 [12]. При данном подходе учитывается только инерционность подвижных элементов излучателя и упругость предварительного поджатия конструкции.

Данная АЧХ (рис. 2) не совпадает с экспериментом по собственным и резонансным частотам, а также по амплитудам ускорения системы. Следовательно, необходимо доработать схему замещения с учетом влияния жесткости корпуса и внутренних колебаний многослойного пьезоактюатора.

В консервативных колебательных системах амплитуды смещения, скорости и ускорения подвижных элементов определяются величинами инерционности и жесткостями системы. Реактивные сопротивления электрической схемы замещения определяются выражениями (1):

$$
X_{\mathrm{m} 1}=j \omega m_{1}, X_{\mathrm{c} 1}=\frac{C_{1}}{j \omega},
$$

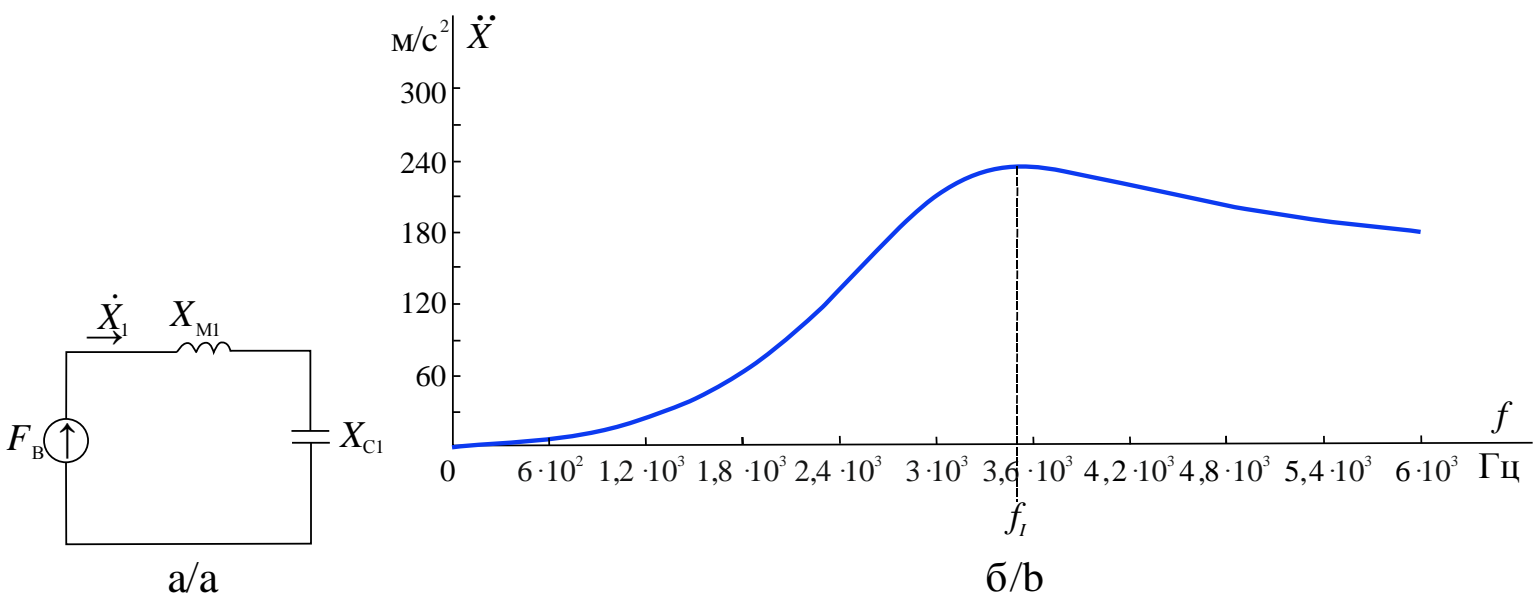

Pис. 2. Результаты расчета по подходу из [12]: а) упрощенная электрическая схема замещения ультразвукового излучателя резонансного типа; б) АЧХ системы

Fig. 2. Results of the calculation from [12]: a) simplified electrical scheme for replacing the ultrasonic emitter; b) amplitude-frequency characteristics system 
где $m_{1}$ - суммарная масса подвижных элементов конструкции (масса толкателя, 1/3 массы многослойного пьезоактюатора, масса двух щёк, двух центраторов), кг; $C_{1}=\frac{1}{k_{1}}-$ жесткость пружины предварительного поджатия, Н/м; $k_{1}$ - упругость пружины предварительного поджатия; $\omega$ - угловая частота вращения, 1/c.

Механическая схема УЗ излучателя представлена на рис. 3 , где $m_{1}$ - суммарная масса подвижных элементов, $m_{2}$ - масса части корпуса со стороны толкателя до смотровых окон, $k_{1}$ - упругость пружины предварительного поджатия, $k_{2}$ - упругость стальных стоек смотровых окон.

В математической модели, кроме подсистемы «толкатель - упругость предварительного поджатия», необходимо учитывать колебательную подсистему «корпус - упругость корпуса». Данный подход позволяет на этапе проектирования рассчитать частотные характеристики УЗ излучателя резонансного типа с учетом деформации корпуса.

Колебания корпуса УЗ излучателя соизмеримы по величине с колебаниями толкателя и при совпадении по фазе, на определенной частоте, дают значительное увеличение суммарных колебаний, что существенно увеличивает электромеханический коэффициент преобразования.

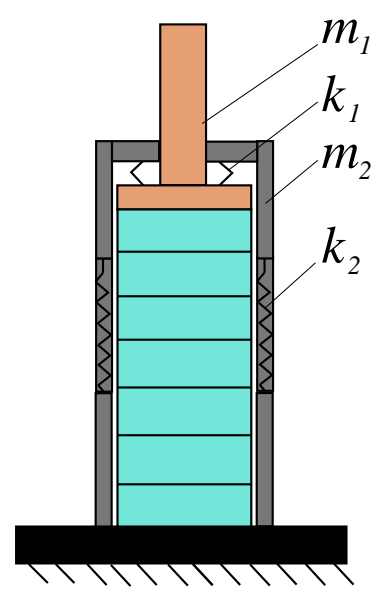

Рис. 3. Механическая схема ультразвукового излучателя резонансного типа с учетом упругой подсистемой корпуса излучателя

Fig. 3. Mechanical scheme of the ultrasonic emitter taking into account the elastic subsystem of the emitter body

Для исследования данной механической колебательной системы использовался формализованный метод электрических аналогий $[1,13,14]$. На рис. 4 представлена эволюция перехода от механической системы к электрической схеме аналогии.
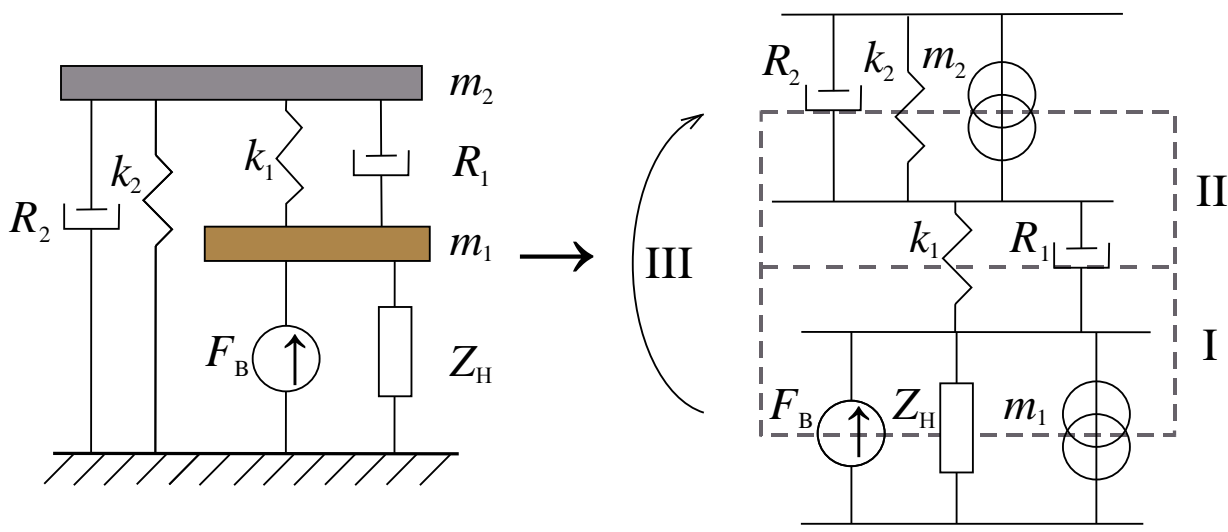

$\mathrm{a} / \mathrm{a}$
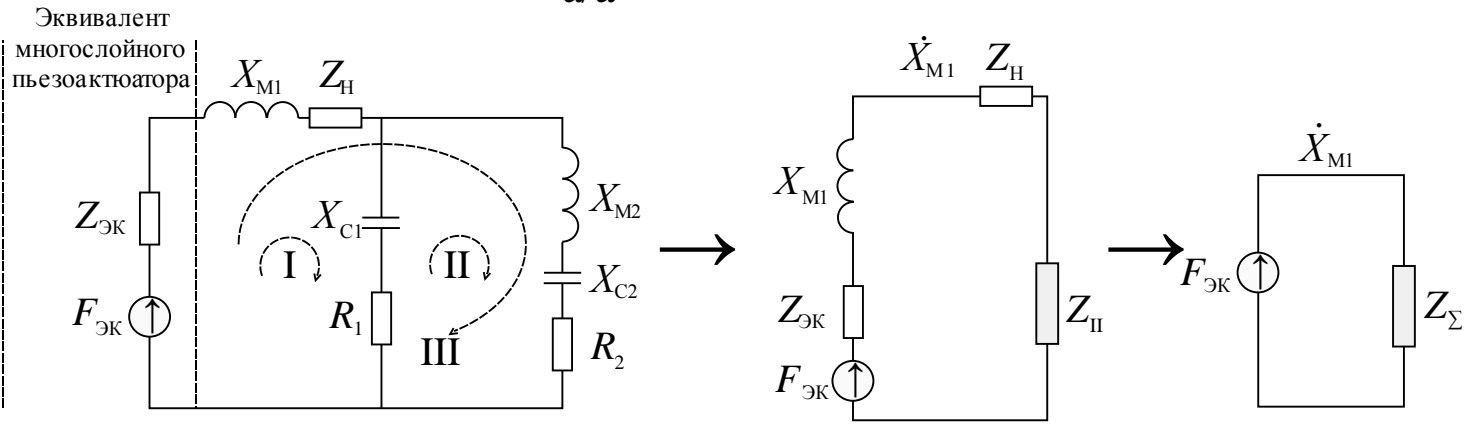

$\sigma / b$

Рис. 4. Преобразование схемы замещения УЗ излучателя резонансного типа в соответствии с [1, 12]: а) преобразование механической схемы замещения излучателя к его трехконтурной электрической схеме замещения; б) преобразование эквивалентной электрической схемы замещения до эквивалентного импеданса

Fig. 4. Conversion of the ultrasonic substitution scheme of the emitter according to [1, 12]: a) conversion of the mechanical scheme of the emitter substitution to its three-circuit electrical substitution scheme; b) conversion of the equivalent electrical replacement scheme to equivalent impedance 
На рис. 4 приняты следующие обозначения: $R_{1}, R_{2}-$ активные сопротивления колебательных контуров I и II, кг/c; $Z_{\text {н }}$ - импеданс нагрузки (на холостом ходу работы УЗ излучателя приравнивается нулю), кг/с; $F_{\mathrm{B}}-$ сила возбуждения многослойного пьезоактюатора, $\mathrm{H}$; Fэк - эквивалентная сила многослойного пьезоактюатора, учитывающая упругие и инерционные сопротивления слоев пьезоактюатора, $\mathrm{H} ; Z_{\text {эк }}$ - эквивалентные внутренние сопротивления (упругие и инерцион- ные сопротивления) многослойного пьезоактюатора, кг/с.

Учет колебательной подсистемы многослойного пьезоактюатора проводится согласно [2, 12, 15-20]. У3 излучатель резонансного типа представляет собой колебательную систему, которую необходимо представлять как единое целое в процессе движения инерционных элементов. Общая электрическая схема замещения представлена на рис. 5.

\section{Многослойный пьезоактюатор}

\section{Механоакустическая} система

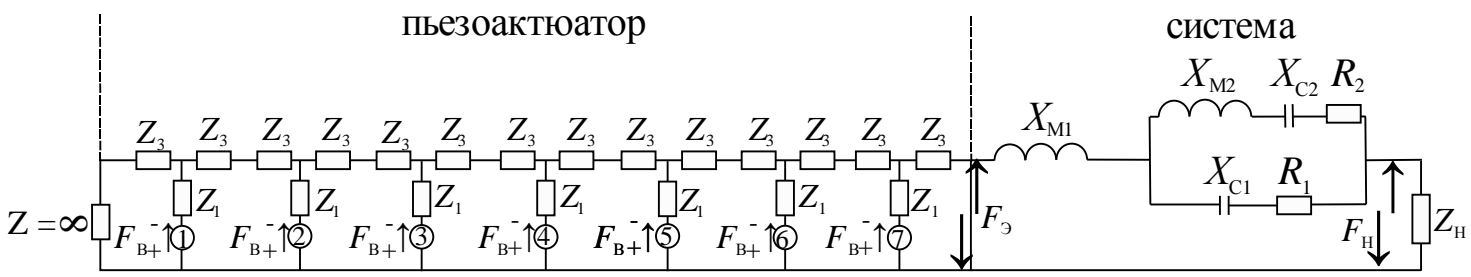

Pис. 5. Общая электрическая схема замещения УЗ излучателя резонансного типа

Fig. 5. General electrical scheme for ultrasonic resonant emitter substitution

Холостой ход УЗ излучателя резонансного типа характеризуется отсутствием сопротивления нагрузки - принимается $Z_{\mathrm{H}}=0$ (рис. 5).

Схема замещения имеет три контура: I, II, III (рис. 4): I - колебательный контур УЗ излучателя; II - колебательный контур части корпуса УЗ излучателя; III внешний колебательный контур, не учитывает жесткость упругости поджатия. Импедансы ответвлений считаются по (2). Каждый контур имеет свою резонансную частоту.

$$
Z_{11}=X_{c 1}+R_{1}, Z_{22}=X_{M 2}+X_{c 2}+R_{2} .
$$

Суммарное сопротивление второго контура определяется из (3):

$$
Z_{I I}=\frac{Z_{11} Z_{22}}{Z_{11}+Z_{22}}
$$

Эквивалентное сопротивление определяется из (4):

$$
Z_{\Sigma}=Z_{\text {эк }}+Z_{I I}+X_{m 1}+Z_{\mathrm{H}} .
$$

Масса подвижных частей подсистемы «толкатель - упругость предварительного поджатия» вычисляется из:

$$
m_{1}=\frac{7 M_{\text {пз }}}{3}+M_{\text {ч }}+M_{\text {ш }}+M_{\text {дс }}+M_{\text {тол }}+M_{\text {ду }},
$$

где $M_{\text {щ }}$ - масса центратора; $M_{\text {щ }}$ - масса щеки; $M_{\text {дс }}$ масса датчика силы; $M_{\text {тол }}$ - масса толкателя; $M_{\text {ду }}$ масса датчика ускорения; $M_{\text {пэ }}$ масса пластины пьезоэлемента.

Масса подвижной части корпуса УЗ излучателя резонансного типа:

$$
m_{2}=V_{\text {кор }} \rho_{f e},
$$

где $V_{\text {кор }}$ - объем подвижной части корпуса излучателя; $\rho_{f e}-$ плотность материала корпуса.

Жесткость стоек окна корпуса излучателя вычисляется по выражению (5):

$$
C_{2}=E \frac{S}{L_{0}} \mathrm{H} / \mathrm{M},
$$

где $E$ - модуль Юнга материала, Па; $S_{\text {c }}$ - суммарная площадь сечения стоек окна излучателя, $\mathrm{m}^{2} ; L_{0}-$ длина окна корпуса излучателя, м.

Резонансные угловые частоты контуров колебательной системы УЗ излучателя ориентировочно определяются из (6):

$$
\omega_{i}=\sqrt{\frac{C_{i}}{m_{i}}}, 1 / \mathrm{c}
$$

где $i=1 \ldots 3 ; C_{i}$ - суммарная жесткость $i$-го контура колебательной системы; $m_{i}$ - суммарная масса $i$-го контура колебательной системы.

В таблице представлены резонансные частоты колебательных контуров конструкции УЗ излучателя резонансного типа.

Таблица. Резонанс контуров УЗ излучателя

Table. Resonance of the ultrasonic emitter circuits

\begin{tabular}{|c|c|}
\hline № контура/Circuit number & $f_{i}, \mathrm{\kappa} \Gamma ц / \mathrm{kHz}$ \\
\hline I & 3,37 \\
\hline II & 26,4 \\
\hline III & 11 \\
\hline
\end{tabular}

Учет движения части корпуса образует в ускорении толкателя ряд резонансныХ мод в АЧХ УЗ излучателя (рис. 6). Без учета колебательной системы многослойного пьезоактюатора.

На рис. 7, a показана схема проведения эксперимента УЗ излучателя резонансного типа. Сигналы с акселерометра AP10 обрабатывались на предварительном усилителе ZET 440 фирмы ZETLAB. Датчик силы представляет собой предварительно прокалиброванный пьезоактюатор (50 слоев).

На осциллографе GDS-72104E фирмы GW Instek снимались сигналы: $U$-напряжения на многослойном пьезоактюаторе, $F$ - сигнал пропорциональный силы многослойного пьезоактюатора, $I$ - ток многослойного пьезоактюатора, $\ddot{X}$ - ускорение толкателя. На рис. 7, б приведена осциллограмма процесса на частоте 20 кГц. 


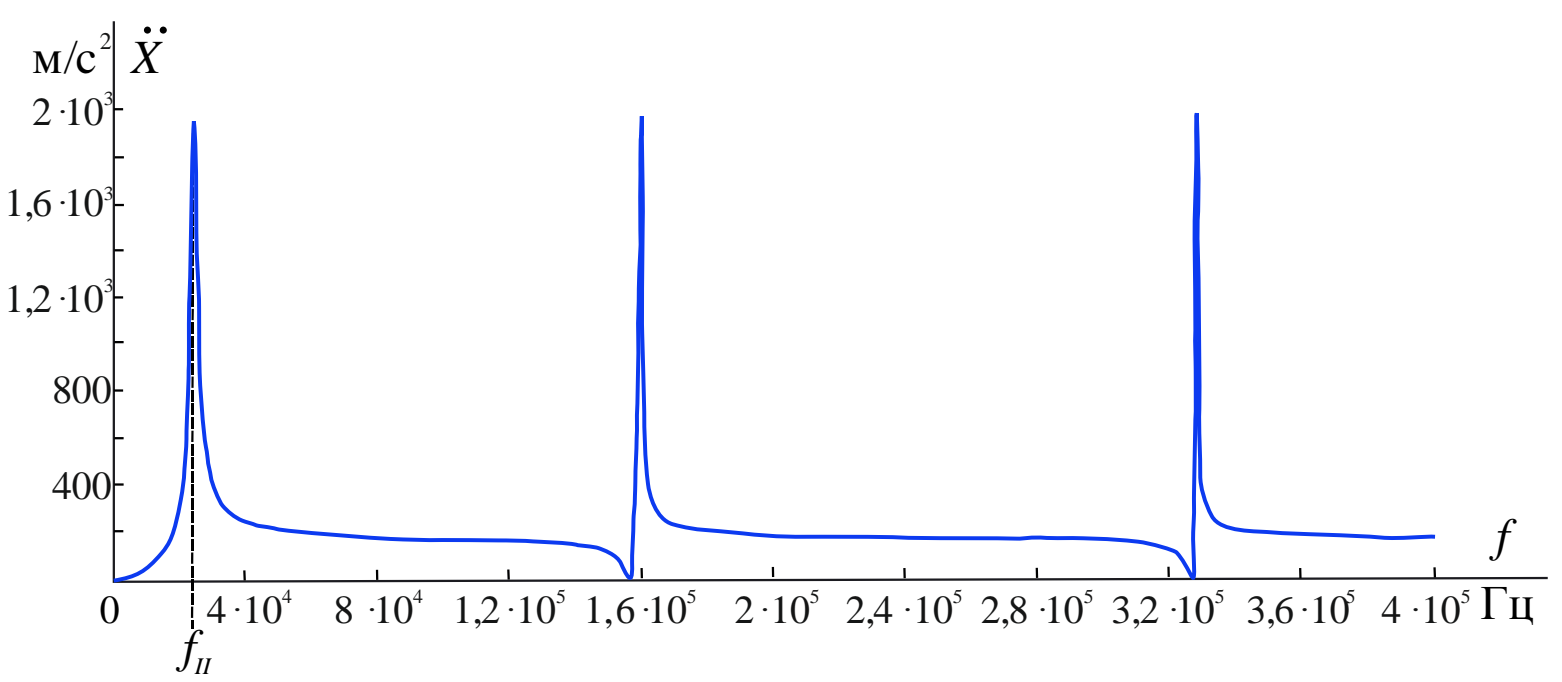

Рис. 6. АЧХ системы УЗ излучателя с учетом участия в движении части корпуса излучателя и без учета колебательных процессов в многослойном пьезоактюаторе

Fig. 6. Amplitude-frequency characteristics system of the ultrasonic emitter taking into account the participation in the motion of the emitter body part and not taking into account oscillation processes in the multilayer piezoactuator

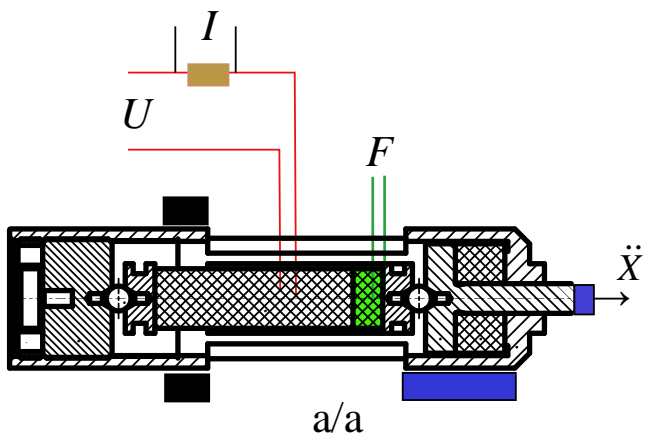

$\mathrm{a} / \mathrm{a}$

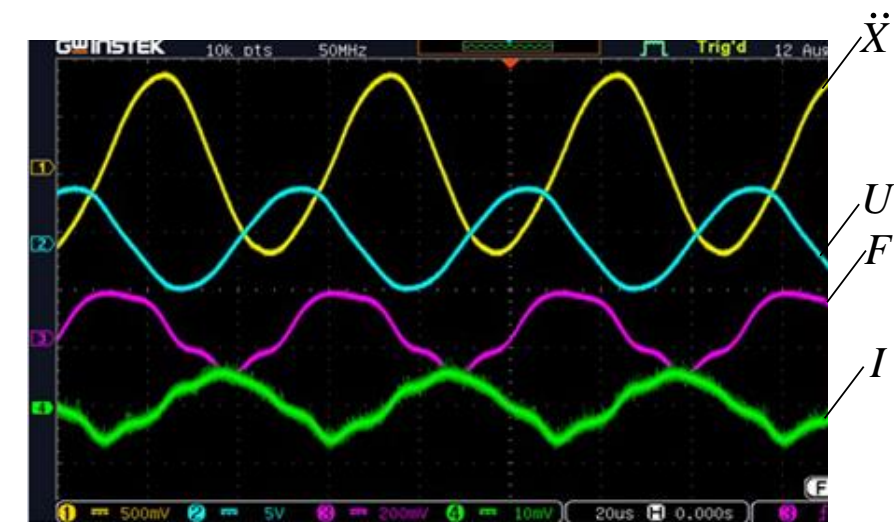

б/b

Pис. 7. Эксперимент: а) схема снятия экспериментальной АЧХ УЗ излучателя при работе на холостом ходу; б) осииллограммы сигналов измерительной системь

Fig. 7. Experiment: a) experimental amplitude-frequency characteristics of ultrasonic emitter while idling; $b$ ) signal oscillograms of the measuring system

На рис. 8, а представлены данные, полученные при эксперименте и при аналитическом расчете с помощью разработанной математической модели. Показано, что основной резонанс по этим характеристикам совпадает и математическая модель адекватна колебательной системе УЗ излучателя. Максимум ускорения толкателя соответствует резонансу колебаний части корпуса, что существенно увеличивает возможности излучателя. На рис. 8, б представлена АЧХ УЗ излучателя для большего диапазона, рассчитанная с помощью разработанной математической модели. На АЧХ УЗ излучателя выявлено несколько резонансов системы, причем есть резонансы с большими ускорениями.

Анализ частотных характеристик, полученных на основе разработанной математической модели, позволяет определить наиболее энергоемкие частоты (резонансы) работы колебательной системы УЗ излучателя резонансного типа.

\section{Заключение}

Анализ результатов проведенного исследования показал, что для получения достоверной информации по работе ультразвукового излучателя резонансного типа при аналитических расчетах необходимо учитывать влияние жесткости корпуса и внутренних колебаний многослойного пьезоактюатора.

Деформации части корпуса ультразвукового излучателя, получаемые за счет наличия смотровых окон, увеличивают ускорение рабочей поверхности толкателя, по сравнению с работой ультразвукового излучателя с абсолютно жестким корпусом, тем самым увеличивается коэффициент преобразования электрической энергии в механическую.

Результаты теоретических расчетов хорошо согласуются с экспериментальными данными, погрешность составляет не более $15 \%$. 

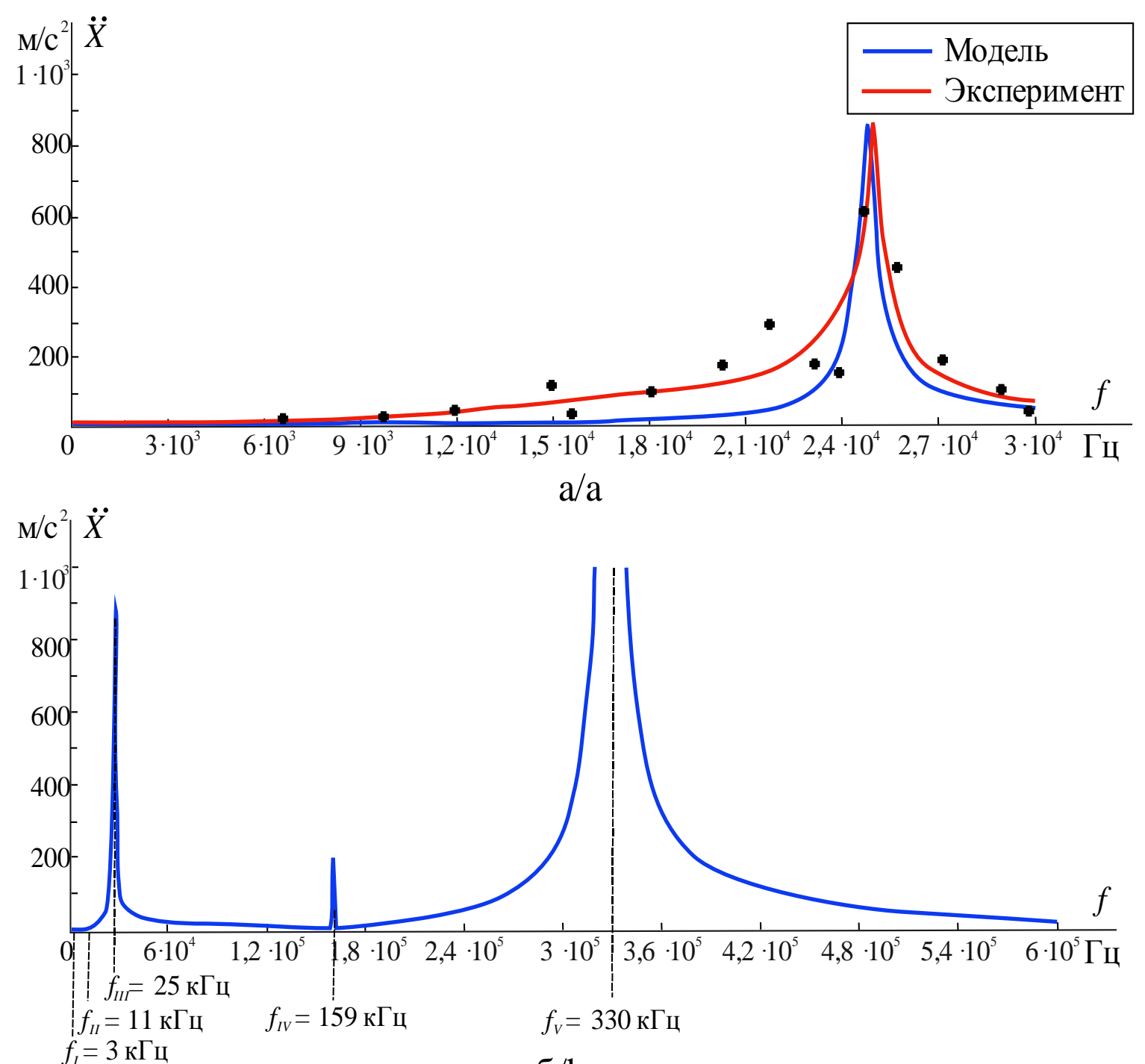

$6 / \mathrm{b}$

Pис. 8. АЧХ УЗ излучателя резонансного типа с учетом влияния многослойного пьезоактюатора и жесткости корпуса: а) сравнение данных расчета и эксперимента; б) данные расчета для большего диапазона частот

Fig. 8. Amplitude-frequency characteristics of ultrasonic emitter, taking into account the influence of the multilayer piezoactuator and the body rigidity: a) comparison of calculation and experiment data; b) calculation data for a larger frequency range

Математическая модель ультразвукового излучателя резонансного типа позволяет определить частоты колебательной системы, на которых наблюдается максимальный коэффициент преобразования энергии, определить необходимую точность настройки частоты источника энергии, рассчитать конструкцию корпуса излучателя по надежности и ресурсу работы.

Разработанная математическая модель ультразвукового излучателя резонансного типа позволяет оценить энергетическое воздействие на любые твердые и

\section{СПИСОК ЛИТЕРАТУРЫ}

1. Математическое моделирование процесса ультразвуковой подготовки высоковязкой нефти к транспорту / А.В. Азин, Е.П. Богданов, С.А. Пономарев, Н.Н. Марицкий, С.В. Рикконен // Известия Томского политехнического университета. Инжиниринг георесурсов. - 2021. - Т. 332. - № 4. - С. 114-122.

2. Зайцев В.В., Кузнецова И.Е. Акустические волны в тонких пьезоэлектрических пластинах. - М.: Радиотехника, 2018. - 240 c. жидкие вещества при выбранном режиме его работы. Таким образом, разработанная математическая модель позволяет определить влияние каждого элемента конструкции ультразвукового излучателя резонансного типа для подготовки нефти и углеводородных топлив к транспорту в условиях Арктики и Антарктики.

Работа выполнена в рамках государственного задания Министерства науки и высшего образования Российской Федерачии (тема № 0721-2020-0036).

3. Gong Jing. Study on deacidification process of waste internal combustion engine oil under the action of ultrasound // IOP Conf. Ser.: Earth Environ. - 2020. - Sci. 467012046

4. Ultrasonic nano-emulsification - a review / M. Seyed, M.-G. Mohsen, G.-G. Roghayeh, M. Massoud, S. Pedram Safarpoura, Z. Majid // Ultrasonics Sonochemistry. -2019 . - 52. - P. 88-105.

5. Xu Y., Langbauer C., Hofstaetter $\mathrm{H}$. The application of ultrasonic technology for cleaning oil contaminated sand // SPE Asia Pacific 
Health, Safety, Security, Environment and Social Responsibility Conference. - Kuala Lumpur, 2017.

6. Гусев В.А., Руденко О.В. Поля радиационных сил и акустические течения в жидком слое на твердом полупространстве // Акустический журнал. - 2019. - Т. 65. - № 2. - С. 166-181.

7. Guz A.N., Bagno A.M. Effect of prestresses on the dispersion of Lamb waves in a system consisting of a viscous liquid layer and a compressible elastic layer // International applied mechanics. 2018. - V. 54 (3). - P. 249-258

8. Huang L., Liang J., Wu C. A three-dimensional indirect boundary integral equation method for modeling elastic wave scattering in a layered half-space // International Journal of Solids and Structures. - 2019. - V. 169. - P. 81-94.

9. Falleta S., Monegato G., Scuderi L. On the discretization and application of two space-time boundary integral equations for 3D wave propagation problems in unbounded domains // Applied Numerical Mathematics. - 2018. - V. 124. - P. 22-43.

10. Intensification of the processes of preparation of drilling and cement mortars using vibration jet activation methods / S.V. Rikkonen, M.A. Dmitrieva, V.N. Leitsin, S.V. Ponomarev, A.V. Azin // IOP Conference Series: Materials Science and Engineering. - 2020. - V. 911. - № 012001. DOI: 10.1088/1757899X/911/1/012001.

11. Способ виброструйной гидродинамической технологии сохранения текучести углеводородных топлив и нефтепродуктов в условиях низких температур / А.В. Азин, Е.П. Богданов, С.В. Пономарев, С.В. Рикконен // Известия Томского политехнического университета. Инжиниринг георесурсов. 2019. - T. 330. - № 4. - C. 41-48.

12. Рикконен С.В., Пономарев С.В., Азин А.В. Моделирование колебательных процессов пьезоэлектрического преобразователя // Вестник Томского государственного университета. Математика и механика. - 2015. - № 2 (34). - С. 86-95.

13. Идентификация параметров механической системы вибрационного электромагнитного активатора по граничным околорезонансным частотам / А.Н. Гаврилин, С.Н. Кладиев, А.С. Глазырин, Е.В. Боловин, В.И. Полищук // Известия Томского по- литехнического университета. Инжиниринг георесурсов. 2019. - T. 330. - № 4. - С. 158-177.

14. Резонансные колебания с предельной амплитудой в вибрационном электромагнитном активаторе / А.Н. Гаврилин, Е.В. Боловин, А.С. Глазырин, С.Н. Кладиев, В.И. Полищук // Известия Томского политехнического университета. Инжиниринг георесурсов. - 2019. -Т. 330. - № 1. - С. 201-213.

15. Thin film flexible/bendable acoustic wave devices: Evolution, hybridization and decoupling of multiple acoustic wave modes / R. Tao, W.B. Wang, J.T. Luo, S.A. Hasan, H. Torun, P. CanyellesPericas, J. Zhou, W.P. Xuan, M.D. Cooke, D. Gibson, Q. Wu, W.P. Ng, J.K. Luo, Y.Q. Fu // Surface \& Coatings Technology. 2019. - V. 357. - P. 587-594

16. Guiding and splitting Lamb waves in coupled-resonator elastic waveguides / Y.-F. Wang, T.-T. Wang, J.-P.Liu, Y.-S. Wang, V. Laude // Composite Structures. - 2018. - V. 206. - P. 588-593.

17. Анисимкин В.И., Воронова Н.В. Особенности генерации нормальных акустических волн высших порядков в тонких пьезоэлектрических пластинах // Акустический журнал. - 2020. T. 66. - № 1. - С. 3-7.

18. Создание эталонной плоской волны ультразвуковой волны в жидкости с помощью плоского пьезоэлектрического преобразователя большого волнового размера / А.А. Крохмаль, Д.А. Николаев, С.А. Цысарь, О.А. Сапожников // Акустический журнал. -2020 . - Т. 66. - № 5. - С. 475-488.

19. Исаев А.Е., Айвазян Ю.М., Поликарпов А.М. Проблемы исследования акустических свойств материалов методами ближнего поля // Альманах современной метрологии. 2020. - № 1. - C. 163-196.

20. Research on the transmission characteristics of air-coupled ultrasound in double-layered bonded structures / X.-G. Wang, W.-L. Wu, Z.-C. Huang, J.-J. Chang, N.-X. Wu // Materials. 2018. - V. 11. - № 310. DOI: 10.3390/ma11020310.

Поступила 15.10.2021 2.

\section{Информация об авторах}

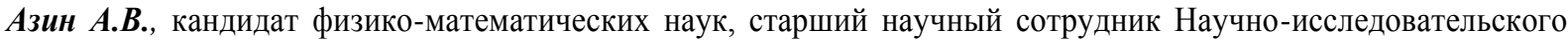
института прикладной математики и механики Томского государственного университета.

Богданов Е.П., кандидат технических наук, доцент Инженерной школы энергетики Национального исследовательского Томского политехнического университета.

Марицкий Н.H., кандидат физико-математических наук, инженер-исследователь Научно-исследовательского института прикладной математики и механики Томского государственного университета.

Пономарев C.A., инженер-исследователь Научно-исследовательского института прикладной математики и механики Томского государственного университета.

Рикконен С.B., кандидат технических наук, доцент, инженер-исследователь Научно-исследовательского института прикладной математики и механики Томского государственного университета. 
UDC 534.21

\title{
MATHEMATICAL MODELING OF A RESONANT-TYPE ULTRASONIC EMITTER FOR PREPARING HIGH-VISCOSITY OIL FOR TRANSPORT
}

\author{
Anton V. Azin'1, \\ antonazin@niipmm.tsu.ru
}

\section{Eugene P. Bogdanov²,} epbogdanov@mail.ru

Nikolay N. Maritsky ${ }^{1}$, nnmar@niipmm.tsu.ru

\section{Sergey A. Ponomarev ${ }^{1}$,} doc.sergeyponomarev@gmail.com

\author{
Sergey V. Rikkonen ${ }^{1}$, \\ rikk2@yandex.ru \\ 1 National Research Tomsk State University, \\ 36, Lenin avenue, Tomsk, 634050, Russia. \\ 2 National Research Tomsk Polytechnic University, \\ 30, Lenin avenue, Tomsk, 634050, Russia.
}

The relevance of this study is caused by the fact that in order to increase the efficiency (productivity) of the ultrasound method for preparing high-viscosity oil for transport it is necessary to take into account all the construction features of the ultrasound emitter in order to fully complete energy matching of the oscillatory system «emitter-load».

The aim of the research is to develop a mathematical model of an ultrasound resonant emitter accounting for the effect of deformation of the emitter body and oscillations of a multilayer piezo actuator on its amplitude-frequency characteristics in order to obtain the total energy characteristics of the system.

Objects: the design of the ultrasound resonant emitter, amplitude-frequency characteristics.

Methods: mathematical modeling of an ultrasound resonant emitter, taking into account the oscillating processes of the movable parts inertia of the structure, the mobility of a emitter body part and the oscillating processes of a multilayer piezo actuator.

Results. The mathematical model makes it possible to calculate the amplitude-frequency characteristics of the oscillatory system accounting for deformations of the ultrasound emitter body and oscillations of a multilayer piezo actuator; to determine the resonant frequencies of the system; to choose the construction of the emitter body and piezo package. Results of the theoretical calculations show very good agreement with experimental data with the deviation of no more than $15 \%$.

Conclusions. The results of the study showed that in order to obtain reliable information on operation of ultrasound resonance emitter, the influence of the rigidity of the body and internal oscillations of the multilayer piezoactuator must be taken into account in the analytical calculations. The deformation of a part of the ultrasound emitter body by the use of inspection windows increases the acceleration of the working pusher as compared to the operation of the radiator ultrasound emitter with a completely rigid body, thereby increasing the coefficient of electrical energy into mechanical one. The mathematical model of the ultrasound resonant emitter makes it possible to determine the oscillation system frequencies at which the maximum energy conversion coefficient is observed and to estimate the necessary accuracy of the energy source frequency setting.

\section{Key words:}

Ultrasonic radiation, emitter, conversion coefficient, energy, thermal fields.

The work was carried out within the framework of the State mission of the Ministry of Science and Higher Education of the Russian Federation (theme 0721-2020-0036).

\section{REFERENCES}

1. Azin A.V., Bogdanov E.P., Ponomarev S.A., Maritsky N.N., Rikkonen S.V. Mathematical modelling of high-viscous oil ultrasonic preparation for transport. Bulletin of the Tomsk Polytechnic University. Geo Assets Engineering, 2021, vol. 332, no. 4. pp. 114-122. In Rus.

2. Zaytsev V.V., Kuznetsova I.E. Akusticheskie volny $v$ tonkikh pezoelektricheskikh plastinakh [Acoustic waves in thin piezoelectric plates]. Moscow, Radiotehnika Publ., 2018. 240 p.

3. Gong Jing. Study on deacidification process of waste internal combustion engine oil under the action of ultrasound. IOP Conf. Ser.: Earth Environ Sci., 2020, vol. 467, no. 012046.

4. Seyed M., Mohsen M.-G., Roghayeh G.-G., Massoud M., Pedram Safarpoura S., Majid Z. Ultrasonic nano-emulsification - a review. Ultrasonics Sonochemistry, 2019, vol. 52, pp. 88-105.
5. Xu Y., Langbauer C., Hofstaetter H. The application of ultrasonic technology for cleaning oil contaminated sand. SPE Asia Pacific Health, Safety, Security, Environment and Social Responsibility Conference. Kuala Lumpur, 2017.

6. Gusev V.A., Rudenko O.V. Polya radiatsionnykh sil i akusticheskie techeniya $\mathrm{v}$ zhidkom sloe na tverdom poluprostranstve [Radiation force fields and acoustic flows in a liquid layer on a solid half-space]. Akusticheskiy zhurnal, 2019, vol. 65 , no. 2 , pp. 166-181.

7. Guz A.N., Bagno A.M. Effect of prestresses on the dispersion of Lamb waves in a system consisting of a viscous liquid layer and a compressible elastic layer. International applied mechanics, 2018, vol. 54 (3), pp. 249-258.

8. Huang L., Liang J., Wu C. A three-dimensional indirect boundary integral equation method for modeling elastic wave scattering in a 
layered half-space. International Journal of Solids and Structures, 2019, vol. 169, pp. 81-94.

9. Falleta S., Monegato G., Scuderi L. On the discretization and application of two space-time boundary integral equations for 3D wave propagation problems in unbounded domains. Applied Numerical Mathematics, 2018, vol. 124, pp. 22-43.

10. Rikkonen S.V., Dmitrieva M.A., Leitsin V.N., Ponomarev S.V., Azin A.V. Intensification of the processes of preparation of drilling and cement mortars using vibration jet activation methods. IOP Conference Series: Materials Science and Engineering, 2020, vol. 911, no. 012001. DOI: 10.1088/1757899X/911/1/012001.

11. Azin A.V., Bogdanov E.P., Ponomarev S.V., Rikkonen S.V. Method of vibro-jet hydrodynamic technology to retain the fluidity of hydrocarbon fuels and petroleum products at low temperatures. Bulletin of the Tomsk Polytechnic University. Geo Assets Engineering, 2019, vol. 330, no. 4, pp. 41-48. In Rus.

12. Rikkonen S., Ponomarev S., Azin A. Simulation of oscillatory processes in a piezoelectric emitter. Tomsk state university journal of mathematics and mechanics, 2015, Iss. 2 (34), pp. 86-95. In Rus.

13. Gavrilin A.N., Kladiev S.N., Glazyrin A.S., Bolovin E.V., Polishchuk V.I. Identification of parameters of vibration electromagnetic activator mechanical system using limiting nearresonance frequency. Bulletin of the Tomsk Polytechnic University. Geo Assets Engineering, 2019, vol. 330, no. 4. pp. 158-177. In Rus.

14. Gavrilin A.N., Bolovin E.V., Glazyrin A.S., Kladiev S.N., Polishchuk V.I. Resonant oscillations with a limiting amplitude in a vibration electromagnetic activator. Bulletin of the Tomsk Polytechnic University. Geo Assets Engineering, 2019, vol. 330, no. 1, pp. 201-213. In Rus.
15. Tao R., Wang W.B., Luo J.T., Hasan S.A., Torun H., CanyellesPericas P., Zhou J., Xuan W.P., Cooke M.D., Gibson D., Wu Q., Ng W.P., Luo J.K., Fu Y.Q. Thin film flexible/bendable acoustic wave devices: Evolution, hybridization and decoupling of multiple acoustic wave modes. Surface\& Coatings Technology, 2019, vol. 357, pp. 587-594.

16. Wang Y.-F., Wang T.-T., Liu J.-P., Wang Y.-S., Laude V. Guiding and splitting Lamb waves in coupled-resonator elastic waveguides. Composite Structures, 2018, vol. 206, pp. 588-593.

17. Anisimkin V.I., Voronova N.V. Osobennosti generatsii normalnykh akusticheskikh voln vysshikh poryadkov $\mathrm{v}$ tonkikh pezoelektricheskikh plastinakh [Features of the generation of higher-order normal acoustic waves in thin piezoelectric plates]. Akusticheskiy zhurnal, 2020, vol. 66, no. 1. pp. 3-7.

18. Krokhmal A.A., Nikolaev D.A., Tsysar S.A., Sapozhnikov O.A. Sozdanie etalonnoy ploskoy volny ultrazvukovoy volny v zhidkosti s pomoshchyu ploskogo pezoelektricheskogo preobrazovatelya bolshogo volnovogo razmera [Creation of a reference plane wave of an ultrasonic wave in a liquid using a flat piezoelectric emitter of large wave size]. Akusticheskiy zhurnal, 2020, vol. 66, no. 5, pp. 475-488.

19. Isaev A.E., Ayvazyan Yu.M., Polikarpov A.M. Problemy issledovaniya akusticheskikh svoystv materialov metodami blizhnego polya [Problems of studying the acoustic properties of materials by near-field methods]. Almanac of modern metrology, 2020, no. 1, pp. 163-196.

20. Wang X.-G., WuW.-L., Huang Z.-C., Chang J.-J., Wu N.-X. Research on the transmission characteristics of air-coupled ultrasound in double-layered bonded structures. Materials, 2018, vol. 11, no. 310. DOI: 10.3390/ma11020310.

Received: 15 October 2021.

\section{Information about the authors}

Anton V. Azin, Cand. Sc., senior researcher, National Research Tomsk State University.

Eugene P. Bogdanov, Cand. Sc., associate professor, National Research Tomsk Polytechnic University.

Nikolay N. Maritsky, Cand. Sc., research engineer, National Research Tomsk State University.

Sergey A. Ponomarev, research engineer, National Research Tomsk State University.

Sergey V. Rikkonen, Cand. Sc., research engineer, National Research Tomsk State University. 